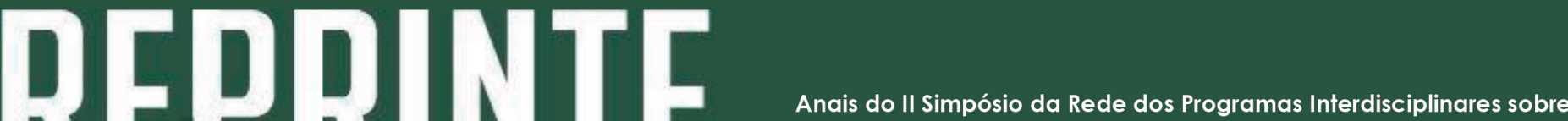

\section{6) Cinesioterapia respiratória para idosos obesos}

Rafael Hideki Abiko'; Braulio Henrique Magnani Branco²; Maria Luiza Amaro Camilo'; Raiane Caroline Garcia'; Marciele Alves Bolognese ${ }^{1}$; Renata Cappellazzo'; Michelle Cardoso Machado dos Santos ${ }^{1}$; Sonia Maria Marques Gomes Bertolini²

\section{Resumo}

A expansibilidade torácica pode ser afetada pelo excesso de gordura corporal. Diante disso, o presente estudo teve como objetivo comparar a capacidade vital forçada (CVF) e o volume expiratório forçado (VEF) no primeiro segundo, antes e após um programa de exercícios respiratórios para idosos obesos. Trata-se de um estudo quase-experimental e longitudinal, que utilizou a cinesioterapia respiratória como método de intervenção em idosos obesos. Os participantes foram avaliados antes e após a intervenção por meio da espirometria. Não houve diferença significativa nos valores de $\operatorname{CVF}(p=0,39) \operatorname{~VEF}_{1}(p=0,53)$, nos momentos pré e pós-intervenção. Baseado nos resultados apresentados, conclui-se que 12 semanas de intervenção de cinesioterapia respiratória não provoca alterações significativas na capacidade pulmonar em idosos obesos.

Palavras-chave: Espirometria. Exercícios respiratórios. Obesidade.

\section{Introdução}

A obesidade é uma pandemia cada vez mais prevalente na população idosa, sendo um problema de saúde pública que preocupa grandemente os governos federal, estadual e municipal (LEBEMBAUM, 2018). Além disso, essa patologia causa algumas repercussões deletérias, como o comprometimento pulmonar (FERREIRA et al, 2017). Nesse aspecto, a gordura corporal em excesso, principalmente na região torácica, diminui a complacência expansibilidade dos pulmões e consequentemente, acaba afetando a capacidade respiratória dos indivíduos (FERREIRA et al, 2017).

Diante disso, a espirometria é um instrumento bastante utilizado para analisar a função pulmonar (FERREIRA et al., 2017). Os achados mais comuns na realização do referido teste é encontrar o volume de reserva expiratório, bem como a capacidade residual funcional reduzi-

1 Mestrandos no Programa de Pós-Graduação em Promoção da Saúde do Centro Universitário de Maringá (Unicesumar). Endereço para correspondência: Centro Universitário de Maringá (Unicesumar), Av. Guedner, 1610, Zona 08, Maringá-PR, Brasil. Email (na ordem em que aparecem): rafaelabiko@gmail.com; malucamiloh_h@hotmail.com; raianercg@gmail.com; mafb-2006@hotmail.com; renatacappellazzo@gmail.com; machado@unicesumar.edu.br

2 Professores do Programa de Pós-Graduação em Promoção da Saúde do Centro Universitário de Maringá (Unicesumar). Email (na ordem em que aparecem): braulio.branco@unicesumar.edu.br; sonia.bertolini@unicesumar.edu.br 
da (TEIXEIRA et al, 2011). Na literatura, nota-se uma lacuna acerca de estudos longitudinais em idosos obesos sem comprometimento pulmonar. Dessa forma, o presente estudo teve como objetivo investigar os efeitos da cinesioterapia respiratória na capacidade vital forçada (CVF) e o volume expiratório forçado (VEF), antes e após 12 semanas de exercícios respiratórios em idosos obesos.

\section{Material e Métodos}

O presente estudo denota um delineamento quase-experimental e longitudinal. Os participantes foram selecionados por amostragem probabilística, por conveniência. Participaram do estudo 19 idosos, com idade igual ou superior a 60 anos, de ambos os sexos, residentes na cidade de Maringá-PR. O estudo foi aprovado pelo Comitê de Ética em Pesquisa (CEP) do Centro Universitário de Maringá (UniCesumar), conforme parecer no 2.596. 181/2018.

Os critérios de inclusão foram: a) residir na cidade local; b) ter idade igual ou superior a 60 anos de idade; c) participação no programa de cinesioterapia respiratória, com no mínimo 75\% de frequência (para serem incluídos nas análises estatísticas). Não foram aceitos: a) participantes que apresentaram algum tipo de distúrbio ou patologia pulmonar que pudesse comprometer as respostas pulmonares ao longo do período de intervenção; b) fumantes; c) participantes incapazes de executar o teste espirométrico de forma correta e d) uso de medicamentos para o tratamento de doenças pulmonares.

O protocolo utilizado no programa de cinesioterapia respiratória consistiu na realização das seguintes etapas, em ordem: 10 minutos de caminhada leve e posteriormente foram executadas de 2 a 3 séries de 10 repetições de pelo menos 3 das técnicas apresentadas a seguir: exercícios de respiração diafragmática, freno labial, diafragmática e freno labial, inspiração fracionada, inspiração fracionada e freno labial, assim como, as referidas técnicas associadas aos exercícios ativos de membros superiores como por exemplo: flexão, extensão, abdução e adução de ombro. Tais atividades foram consumadas duas vezes por semana, no período da manhã, as segundas e quartas-feiras, com duração aproximada de 30 minutos.

Para analisar os volumes respiratórios utilizou-se o espirômetro interpretativo Spirostik (Geratherm). O idoso (a) foi posicionado sentado de forma ereta e recebia a instrução sobre os procedimentos técnicos da medida, após as explanações para execução do teste, foi colocado pelos pesquisadores um prendedor nasal no nariz do avaliado (a); subsequentemente, foi dado o comando verbal ao (a) idoso (a) para realização da medida; os (as) idosos (as) repetiram o teste até serem registradas dois testes reprodutíveis, sendo considerado o melhor teste para anotação das variáveis, nas quais foram seguidas as Diretrizes da European Respiratory Society e da American Thoracic Society (MILLER et al, 2005). Os testes foram realizados no Laboratório Interdisciplinar de Intervenção em Promoção da Saúde (LIIPS) da Unicesumar, em ambiente climatizado com temperatura controlada $\left(24^{\circ} \mathrm{C}\right)$.

A normalidade dos dados foi testada por meio do teste de Shapiro-Wilk. Após a referida confirmação, os dados foram apresentados pela média e desvio padrão ( \pm ). Utilizou-se o teste $t$ pareado para a comparação dos momentos (pré e pós-intervenção). Adotando um nível de significância de 5\%. Todas as análises foram conduzidas mediante ao pacote estatístico SPSS 22.0. 


\section{Resultados}

Participaram do estudo 15 mulheres e 4 homens. A caracterização da amostra está descrita na tabela 1.

Tabela 1. Caracterização da amostra do estudo.

\begin{tabular}{l|c}
\multicolumn{1}{c|}{ Variáveis } & Média \pm desvio padrão \\
\hline Idade (anos) & $66,76 \pm 4,44$ \\
Peso corporal $(\mathrm{kg})$ & $88,7 \pm 15,32$ \\
$\mathrm{IMC}\left(\mathrm{kg} / \mathrm{m}^{2}\right)$ & $34,12 \pm 5,04$ \\
\hline
\end{tabular}

Nota: IMC = índice de massa corporal $\left(\mathrm{kg} / \mathrm{m}^{2}\right)$.

Fonte: elaborado pelos autores.

A tabela 2 apresenta o resultado das médias dos valores da CVF e VEF1 pré e pós-cinesioterapia respiratória.

Tabela 2. Valores espirométricos de $\mathrm{CVF}_{\text {e }} \mathrm{VEF}_{1}$ nos momentos pré e pós 12 semanas de cinesioterapia respiratória.

\begin{tabular}{l|c|c|c}
\multicolumn{1}{c|}{ Variáveis } & $\begin{array}{c}\text { Pré } \\
\text { Média } \pm \text { desvio padrão }\end{array}$ & Média \pm desvio padrão & $\boldsymbol{P}$ \\
\hline CVF & $2,45 \pm 0,70$ & $2,38 \pm 0,83$ & 0,39 \\
VEF $_{1}$ & $2,20 \pm 0,55$ & $2,15 \pm 0,51$ & 0,53 \\
\hline Nota: $C V F=$ Capacidade vital forçada e $\mathrm{VEF}_{1}=$ Volume expiratório forçado no primeiro segundo.
\end{tabular}

Fonte: elaborado pelos autores.

De acordo com as respostas verificadas na tabela 2, não foram observadas diferenças significativas para as comparações dos valores de CFV e $\mathrm{VEF}_{1}$ mensurados antes e após o período de intervenção.

Em conformidade com Ferreira et al (2017), a espirometria é uma avaliação que depende do esforço do paciente, o qual deve ter o entendimento da técnica e igualmente, cooperar para obtenção correta da medida. Qualquer circunstância negativa, como cansaço no dia do teste e falta de motivação para realização da avaliação, pode ser descrita como fator interveniente que provavelmente, irá subestimar a obtenção da medida. Portanto, durante a condução de testes, principalmente com o público idoso, a reprodutibilidade das medidas deve ser testada, a fim de proporcionar maior confiabilidade aos dados coletados. Por outro lado, ressalta-se que o equipamento utilizado é considerado como padrão-ouro de medida. Por fim, salienta-se que as coletas foram concretizadas por profissionais altamente treinados e capacitados.

\section{Considerações Finais}

Conclui-se que o estudo de 12 semanas de intervenção com cinesioterapia respiratória não provoca alterações significativas na capacidade pulmonar de idosos obesos. Em vista disso, sugere-se a realização de estudos mais prolongados e/ou com maior frequência semanal. 


\section{Respiratory kinesiotherapy for obese elderly people}

\section{Abstract}

Thoracic expandability can be affected by excess body fat. Therefore, the present study aimed to compare forced vital capacity (FVC) and forced expiratory volume (FEV) in the first second before and after a respiratory exercise program for obese elderly patients. It is a quasi-experimental and longitudinal study, which used respiratory kinesiotherapy as a method of intervention in obese elderly people. Participants were assessed before and after the intervention using spirometry. There was no significant difference in the values of FVC $(p=0.39)$ and FEV1 $(p=0.53)$, both before and after intervention. Based on the results presented, it is concluded that 12 weeks of intervention of respiratory kinesiotherapy does not cause significant changes in lung capacity in obese elderly.

Keywords: Spirometry. Respiratory exercises. Obesity.

\section{Referências}

FERREIRA, M. S. et al. Espirometria e capnografia volumétrica na avaliação da função pulmonar de indivíduos obesos e eutróficos sem asma. Jornal de Pediatria. v. 93, n. 4, p. 398- 405, 2017.

LEBEMBAUM, M. et al. Development and validation of a population based risk algorithm for obesity: The Obesity Population Risk Tool (OPoRT). v. 13, n. 1, p. 1- 11, 2018.

MILLER, M. R. et al. Standardisation of spirometry. European Respiratory Journal. v. 26, p. 319- 338, 2005.

TEIXEIRA, A. B. et al. Influência da Posição na Espirometria de Pacientes Obesas Grau III. Revista Brasileira de Anestesiologia. v. 61, n. 6, p. 713- 719, 2011. 